\title{
A new k-epsilon model consistent with Monin-Obukhov similarity theory
}

van der Laan, Paul; Kelly, Mark C.; Sørensen, Niels N.

Published in:

Wind Energy

Link to article, DOI:

10.1002/we.2017

Publication date:

2017

Document Version

Peer reviewed version

Link back to DTU Orbit

Citation (APA):

van der Laan, P., Kelly, M. C., \& Sørensen, N. N. (2017). A new k-epsilon model consistent with Monin-Obukhov similarity theory. Wind Energy, 20(3), 479-489. https://doi.org/10.1002/we.2017

\section{General rights}

Copyright and moral rights for the publications made accessible in the public portal are retained by the authors and/or other copyright owners and it is a condition of accessing publications that users recognise and abide by the legal requirements associated with these rights.

- Users may download and print one copy of any publication from the public portal for the purpose of private study or research.

- You may not further distribute the material or use it for any profit-making activity or commercial gain

- You may freely distribute the URL identifying the publication in the public portal

If you believe that this document breaches copyright please contact us providing details, and we will remove access to the work immediately and investigate your claim 


\title{
A new k-epsilon model consistent with Monin-Obukhov similarity theory
}

\author{
M. Paul van der Laan, Mark C. Kelly and Niels N. Sørensen \\ Department of Wind Energy, Technical University of Denmark, Risø campus, DK-4000 Roskilde, Denmark.
}

\begin{abstract}
A new $k-\varepsilon$ model is introduced that is consistent with Monin-Obukhov similarity theory (MOST). The proposed $k-\varepsilon$ model is compared with another $k-\varepsilon$ model that was developed in an attempt to maintain inlet profiles compatible with MOST. It is shown that the previous $k-\varepsilon$ model is not consistent with MOST for unstable conditions, while the proposed $k-\varepsilon$ model can maintain MOST inlet profiles over distances of $50 \mathrm{~km}$. Copyright (c) 2016 John Wiley \& Sons, Ltd.
\end{abstract}

\section{KEYWORDS}

$k-\varepsilon$ eddy viscosity model; atmospheric stability; Monin-Obukhov similarity theory; CFD; RANS.

Correspondence

M. Paul van der Laan, Department of Wind Energy, Technical University of Denmark, Risø campus, DK-4000 Roskilde, Denmark.

Email: plaa@dtu.dk.

Received 28 September 2015; Revised 30 May 2016; Accepted 8 July 2016

\section{INTRODUCTION}

Atmospheric stability is important for wind energy because it influences the characteristics of the atmospheric boundary layer $(\mathrm{ABL})$ in which wind turbines are operating. In stable conditions, buoyancy forces suppress turbulence and thus vertical fluxes, subsequently increasing vertical shear, while decreasing the ABL depth. The low ambient turbulence delays wind turbine wake recovery, which can increase losses in annual energy production of wind farms due to wake interaction. When the atmosphere is unstable, the buoyancy forces increase the boundary layer height, and the ambient turbulence levels are enhanced. Both the increased vertical shear in stable conditions and the high ambient turbulence levels in unstable conditions can increase the blade fatigue loads. Moreover, measurements and simulations of the diurnal cycle on land show that the ABL is non-neutral most of the day.[1] In addition, measurements of offshore wind farms where seasonal variations are more pronounced than daily variations - indicate that for the low wind speed range (i.e. less than $15 \mathrm{~m} \mathrm{~s}^{-1}$ ), non-neutral atmospheric stability is prevailing as well.[2] It is, therefore, important to include atmospheric stability in wind turbine simulations involving loads and annual energy production.

The atmospheric stability of the atmospheric surface layer (ASL) can be represented by Monin-Obukhov similarity theory (MOST) [3]. MOST describes velocity, temperature and turbulence profiles of the ASL for neutral and non-neutral conditions, using theoretical considerations and emperically fitted parameters. In the present work, we are interested in applying MOST to Reynolds-averaged Navier-Stokes (RANS), where the turbulence is modeled with the $k$ - $\varepsilon$ model. However, it is well known that MOST profiles are not in balance with the turbulent transport equations of the $k-\varepsilon$ model in their standard configuration. [4, 5] This means that the vertical profiles inserted at inlet will develop downstream, and the flow solution (e.g. a wind turbine wake) will be dependent on the distance between the inlet and the area of interest (e.g. a wind turbine). Several authors have proposed changes of the turbulent transport equations, in order to make the $k$ - $\varepsilon$ model consistent with MOST, mainly for stable atmospheric conditions. These changes are often parameterizations of one or two model constants, as listed in Table I. Freedman and Jacobson[4] argued that the $k$-equation is in near equilibrium in stable atmospheric conditions and changes only need to be made in the $\varepsilon$-equation. Freedman and Jacobson proposed to introduce a $C_{\varepsilon, 1}$ as a function of the flux Richardson number to overcome the inconsistency with the $\varepsilon$-equation in a stable ASL. In addition, Freedman and Jacobson used an eddy-viscosity coefficient $C_{\mu}$ that is also a function of the flux Richardson number, although this is not necessary for the purpose of sustaining MOST profiles. Alinot and Masson[5, 6] proposed 
to modify the transport equation by introducing a $C_{\varepsilon, 3}$ as function of the stability parameter $\zeta$, for stable and unstable atmospheric conditions. In addition, Alinot[6] redefined the inlet profile for $\varepsilon$ to account for the imbalance with the $k$ equation, and showed that the method works for small domains. Unfortunately, the method does not work for large domains because the transport equation for turbulent kinetic energy (TKE) $k$ is still not in equilibrium with MOST, especially in unstable atmospheric conditions, which will be shown in the present paper. Parente et al.[7] proposed to add a source term in the $k$-equation such that the $k-\varepsilon$ model can sustain a $k$ inlet profile that is variable with height. We will combine the ideas of Parente et al.[7], and Alinot and Masson[5,6] into a new method where an additional analytical source term in the $k$-equation is added and a variable $C_{\varepsilon, 3}$ is used, such that stable and unstable MOST profiles are maintained over a long distance. In theory, one can also choose to parameterize other constants in the $\varepsilon$-equation, however, it is more natural to parameterize $C_{\varepsilon, 3}$ because this parameter does not exist in the $k-\varepsilon$ model for neutral conditions. In addition, the new method is applicable for any MOST profile because it is not restricted to a particular choice of constants.

The description of MOST and corresponding assumptions are summarized in Section 2. The turbulence modeling of a neutral and non-neutral ASL using MOST is described in Section 3. Section 3 also discusses the new method for maintaining MOST profiles in large domains. The simulations are discussed in Section 4.

\section{MONIN-OBUKHOV SIMILARITY THEORY}

The decription and assumptions of MOST can be found in the literature, e.g. Panofsky and Dutton [8], and Monin and Yaglom [9]. For completeness of the present work, an overview is presented here.

MOST assumes that the ASL is steady state and horizontally homogenous. In addition, the turbulent stresses $\overline{u^{\prime} w^{\prime}}$ and $\overline{w^{\prime} \theta^{\prime}}$ (also known as vertical turbulent heat flux) are assumed to be constant with height. Note that $U$ is the streamwise velocity (in $x$-direction), $W$ is the vertical velocity normal to the ground ( $z$-direction) and $\theta$ is the potential temperature; here, we use capital letters to denote mean values, and lower case indicates deviations from the corresponding mean value. Furthermore, MOST includes the assumption that the normalized velocity and potential temperature gradients can be described by analytical functions $\left(\Phi_{m}(\zeta)\right.$ and $\Phi_{h}(\zeta)$, respectively) that are dependent on a single stability parameter $\zeta$,

$$
\zeta \equiv \frac{z}{L}=-\frac{\kappa z}{u_{*}^{3}} \frac{g}{\theta_{0}} \overline{w^{\prime} \theta^{\prime}}
$$

All these assumptions can be summarized as follows:

$$
\begin{aligned}
\overline{u^{\prime} w^{\prime}} & =\text { const. }=-u_{*}^{2}, \\
\overline{w^{\prime} \theta^{\prime}} & =\text { const. }=-u_{*} \theta_{*}, \\
\frac{\kappa z}{u_{*}} \frac{\partial U}{\partial z} & =\Phi_{m}(\zeta), \\
\frac{\kappa z}{\theta_{*}} \frac{\partial \theta}{\partial z} & =\Phi_{h}(\zeta),
\end{aligned}
$$

where $u_{*}$ is the friction velocity, $\theta_{*}$ is the surface-layer temperature scale, $L$ is the Monin-Obukhov length, $g$ is the gravitational acceleration $\left(9.8 \mathrm{~m} \mathrm{~s}^{-2}\right), \kappa$ is the von Kármán constant [10], and $\theta_{0}$ is the potential temperature in the ASL. For $\zeta \rightarrow 0$, the assumptions of MOST also lead to the neutral ASL solution, with $\Phi_{m}(0)=\Phi_{h}(0)=1$, where both the heat flux and the gradient of the potential temperature are zero.

The Boussinesq approximation [11] is used to parameterize the deviatoric Reynolds stresses [12]

$$
\overline{u_{i}^{\prime} u_{j}^{\prime}}-\frac{2}{3} k \delta_{i j}=-\nu_{T}\left(\frac{\partial U_{i}}{\partial x_{j}}+\frac{\partial U_{j}}{\partial x_{i}}\right)
$$

where $\delta_{i j}$ is the Kronecker delta, $\partial U_{i} / \partial x_{j}$ are the mean velocity gradients, $k \equiv \overline{u_{k}^{\prime} u_{k}^{\prime}} / 2$ is the TKE and $\nu_{T}$ is the turbulent eddy viscosity. From equation (6) and the assumption of equation (4), it follows that the turbulent eddy viscosity $\nu_{T}$ can be written as

$$
\nu_{T}=\frac{-\overline{u^{\prime} w^{\prime}}}{\partial U / \partial z}, \quad \text { or } \quad \nu_{T_{\mathrm{MO}}}=\frac{\kappa u_{*} z}{\Phi_{m}(\zeta)}
$$

since all other velocity gradients are assumed to be zero. Note that the subscript MO denotes the viscosity conforming to MOST. The classical MOST profiles of mean velocity and potential temperature are then found by integration of equations 
(4) and (5)

$$
\begin{array}{cc}
U(z)=\frac{u_{*}}{\kappa}\left[\ln \left(\frac{z}{z_{0}}\right)-\Psi_{m}(\zeta)\right], & \Psi_{m}(\zeta)=\int_{z_{0} / L}^{z / L}\left[1-\Phi_{m}(\zeta)\right] d \ln \zeta \\
\Theta(z)-\theta_{0}=\frac{\theta_{*}}{\kappa}\left[\ln \left(\frac{z}{z_{0}}\right)-\Psi_{h}(\zeta)\right], & \Psi_{h}(\zeta)=\int_{z_{0} / L}^{z / L}\left[1-\Phi_{h}(\zeta)\right] d \ln \zeta
\end{array}
$$

where $\theta_{0}$ is the potential temperature near the surface (i.e. at $z=z_{0}$ ) and $\theta_{*}$ can be written as:

$$
\theta_{*}=\frac{\theta_{0} u_{*}^{2}}{g \kappa L},
$$

using equations (1) and (3). Note that the terms with $z_{0} / L$ are neglected in equations (8) and (9). Field measurements of the ASL suggest the following analytical functions $\Phi_{m}$ and $\Phi_{h}$ in stable and unstable stratification [13, 14]:

$$
\begin{array}{lll}
\text { Unstable: } & \Phi_{m}=\left(1-\gamma_{1} \zeta\right)^{-1 / 4}, & \Psi_{m}=\ln \left[\frac{1}{8}\left(1+\Phi_{m}^{-2}\right)\left(1+\Phi_{m}^{-1}\right)^{2}\right]-2 \arctan \left(\Phi_{m}^{-1}\right)+\frac{\pi}{2}, \\
(-2<\zeta<0) & \Phi_{h}=\sigma_{\theta}\left(1-\gamma_{2} \zeta\right)^{-1 / 2}, & \Psi_{h}=\left(1+\sigma_{\theta}\right) \ln \left[\frac{1}{2}\left(1+\Phi_{h}^{-1}\right)\right]+\left(1-\sigma_{\theta}\right) \ln \left[\frac{1}{2}\left(-1+\Phi_{h}^{-1}\right)\right], \\
\text { Stable: } & \Phi_{m}=1+\beta \zeta, & \Psi_{m}=-\beta \zeta, \\
(0<\zeta<1) & \Phi_{h}=\sigma_{\theta}+\beta \zeta, & \Psi_{h}=\left(1-\sigma_{\theta}\right) \ln (\zeta)-\beta \zeta,
\end{array}
$$

where $\Psi_{m}$ and $\Psi_{h}$ follow from integration as defined in equations (8) and (9); other similar analytical forms for these functions have also been reported (e.g. [15]). We remind that, here, $\phi^{-1}$ denote reciprocals, (not inverse functions). ${ }^{*}$ The constants $\beta, \gamma_{1}, \gamma_{2}$ and the turbulent Prandtl number $\sigma_{\theta}$ are empirically determined via measurements; commonly-used values of these constants are

$$
\begin{array}{lllll}
\text { Businger } \text { et al. (Kansas experiment) [13]: } & \sigma_{\theta}=0.74, & \beta=4.7, & \gamma_{1}=15, & \gamma_{2}=9, \\
\text { Dyer [14]: } & \sigma_{\theta}=1, & \beta=5, & \gamma_{1}=16, & \gamma_{2}=16 .
\end{array}
$$

The similarity functions in equation (11) are written without $(\zeta)$; for brevity, we will use such abbreviation hereafter.

If one writes the rate equation for TKE in non-dimensionalized form, then it is possible to relate $\Phi_{m}$ to analogous MOST functions for TKE components (e.g. [16]). More specifically, normalizing the TKE budget

$$
T+\mathcal{P}+B=\varepsilon,
$$

by the surface-layer dissipation rate $u_{*}^{3} /(\kappa z)$, one may write

$$
\frac{\kappa z}{u_{*}^{3}}(T+\mathcal{P}+B)=\Phi_{T}+\Phi_{m}+\Phi_{B}=\Phi_{\varepsilon}
$$

where the normalized dissipation is defined as $\Phi_{\varepsilon} \equiv \varepsilon \kappa z / u_{*}^{3}$. In equation (14), $T$ represents transport of TKE (with $\Phi_{T}$ as its normalized form), and

$$
\mathcal{P} \equiv-\overline{u_{i}^{\prime} u_{j}^{\prime}} \frac{\partial U_{i}}{\partial x_{j}}
$$

is the turbulent production due to shear, which, in horizontally homogeneous conditions, can be simplified to

$$
\mathcal{P}=-\overline{u^{\prime} w^{\prime}} \frac{\partial U}{\partial z}=\nu_{T}\left(\frac{\partial U}{\partial z}\right)^{2}=\frac{u_{*}^{3}}{\kappa z} \Phi_{m},
$$

using equations (4) and (7). $B$ is the rate of turbulent production or destruction of TKE because of buoyancy

$$
B \equiv \frac{g}{\theta_{0}} \overline{w^{\prime} \theta^{\prime}}=\frac{u_{*}^{3}}{\kappa z} \Phi_{B}=-\frac{u_{*}^{3}}{\kappa z} \zeta
$$

\footnotetext{
* We also note that the function $\Psi_{h}$ in equation (11) is only well-defined for $\zeta \rightarrow 0$ when $\sigma_{\theta}=1$, since $\sigma_{\theta}=\phi_{h} / \phi_{m}$ following the Boussinesq approximation for vertical fluxes. While $\sigma_{\theta} \neq 1$ is sometimes chosen in the literature, in such cases typically the contributions to $\Psi_{h}$ with coefficient $\left(1-\sigma_{\theta}\right)$ are neglected in equation (11).
} 
where the right-most expression follows from (1). Note that generally the virtual potential temperature $\theta_{v}$ should be used in equation (17); however, we are not considering water vapor in the present work, thus e.g. $\theta_{v, 0}=\theta_{0}$. For the normalized turbulent dissipation rate $\left(\Phi_{\varepsilon} \equiv \varepsilon \kappa z / u_{*}^{3}\right)$, a commonly used parameterization is Panofsky and Dutton's [8] relation

$$
\Phi_{\varepsilon}=\left\{\begin{array}{ll}
1-\zeta & \zeta<0 \\
\Phi_{m}-\zeta & \zeta>0
\end{array} .\right.
$$

Note that forms for $\Phi_{\varepsilon}$ can contain implicit assumptions that are potentially inconsistent with equation (14); for example equation (18) implies small buoyant production in unstable conditions and neglects turbulent transport stable conditions. The latter assumptions, however, eliminate the need for a form for $\Phi_{T}$ in the dimensionless TKE budget.

\section{TURBULENCE MODELING OF NON-NEUTRAL ASL USING MOST}

The standard $k-\varepsilon$ turbulence model from Launder and Spalding [17] is popular in RANS simulation of atmospheric flows. This 2-equation turbulence model predicts only isotropic Reynolds stresses $\left(\overline{u^{\prime} u^{\prime}}=\overline{v^{\prime} v^{\prime}}=\overline{w^{\prime} w^{\prime}}\right)$, because the Boussinesq approximation of equation (6) is used with a scalar turbulent eddy viscosity; the latter is defined as

$$
\nu_{T} \equiv C_{\mu} \frac{k^{2}}{\varepsilon},
$$

with $C_{\mu}$ as a constant and $\varepsilon$ as the rate of turbulent dissipation. Using equation (19) and the MOST profile from equation (7), the turbulent dissipation of equation (14) then implies a vertical TKE profile, i.e.

$$
k(z)=\left(\frac{\nu_{T_{M O}} \varepsilon}{C_{\mu}}\right)^{1 / 2}=\frac{u_{*}^{2}}{\sqrt{C_{\mu}}}\left(\frac{\Phi_{\varepsilon}}{\Phi_{m}}\right)^{1 / 2} .
$$

The standard transport equations for $k$ and $\varepsilon$ can be written as [18]

$$
\frac{D k}{D t}=\mathcal{D}_{k}+\mathcal{P}-\varepsilon+B, \quad \frac{D \varepsilon}{D t}=\mathcal{D}_{\varepsilon}+\left(C_{\varepsilon, 1} \mathcal{P}-C_{\varepsilon, 2} \varepsilon+C_{\varepsilon, 3} B\right) \frac{\varepsilon}{k},
$$

where $\mathcal{D}_{k}$ and $\mathcal{D}_{\varepsilon}$ represent the transport of $k$ and $\varepsilon$ through diffusion, respectively

$$
\mathcal{D}_{k} \equiv \nabla \cdot\left(\frac{\nu_{T}}{\sigma_{k}} \nabla k\right), \quad \mathcal{D}_{\varepsilon} \equiv \nabla \cdot\left(\frac{\nu_{T}}{\sigma_{\epsilon}} \nabla \varepsilon\right) .
$$

In equation (21), the kinematic molecular viscosity $\nu$ is neglected, since the flow of interest is characterized by a high Reynolds number and consequently $\nu<<\nu_{T}$. The parameters $C_{\mu}, C_{\varepsilon, 1}, C_{\varepsilon, 2}, C_{\varepsilon, 3}, \sigma_{k}, \sigma_{\varepsilon}$ in equations (21) and (22) are listed in Table I, for 3 different $k-\varepsilon$ models used to model atmospheric flows. The $k-\varepsilon$ MOST model of Alinot and Masson and our proposed $k-\varepsilon$ MOST model are further discussed in Sections 3.1.2 and 3.1.3, respectively.

Table I. Model constants of different $k-\varepsilon$ models, used for atmospheric flows.

\begin{tabular}{lcccccccc}
\hline & $C_{\mu}$ & $\kappa$ & $C_{\varepsilon, 1}$ & $C_{\varepsilon, 2}$ & $C_{\varepsilon, 3}$ & $\sigma_{k}$ & $\sigma_{\varepsilon}$ & $\sigma_{\theta}$ \\
\hline$k-\varepsilon$ ASL neutral (Sørensen[19]) & 0.03 & 0.40 & 1.21 & 1.92 & 0 & 1.00 & 1.30 & - \\
$k-\varepsilon$ MOST (Alinot and Masson[5]) & 0.033 & 0.42 & 1.176 & 1.92 & eq. (38) & 1.00 & 1.30 & 1 \\
$k-\varepsilon$ MOST proposed & 0.03 & 0.40 & 1.21 & 1.92 & eq. (46) & 1.00 & 1.30 & 1 \\
\hline
\end{tabular}

As proposed by Richards and Hoxey[20], the constant $C_{\mu}$ is based on atmospheric measurements that are summarized by Panofsky and Dutton[8], and $C_{\varepsilon, 1}$ is adapted to maintain the logarithmic solution via [18] in neutral conditions,

$$
C_{\mu}^{1 / 2} \sigma_{\varepsilon}\left(C_{\varepsilon, 2}-C_{\varepsilon, 1}\right)=\kappa^{2}
$$

Equation (23) is derived by substitution of the neutral ASL solution

$$
U(z)=\frac{u_{*}}{\kappa} \ln \left(\frac{z}{z_{0}}\right), \quad k=\frac{u_{*}^{2}}{\sqrt{C_{\mu}}}, \quad \varepsilon(z)=\frac{u_{*}^{3}}{\kappa z},
$$

into the $k$ - $\varepsilon$ equations (21). When equation (23) holds, the neutral ASL solution of equation (24) is in equilibrium with the RANS equations - in combination with the standard $k-\varepsilon$ model and the correct boundary conditions, as discussed in Section 4.1.2. 
Using equations (3), (5) and (7), the vertical turbulent heat flux $\overline{w^{\prime} \theta^{\prime}}$ can be determined from

$$
\overline{w^{\prime} \theta^{\prime}}=-\frac{\nu_{T}}{\sigma_{\theta}} \frac{\partial \theta}{\partial z}
$$

where we set the turbulent Prandtl number $\sigma_{\theta}$ as[13]

$$
\sigma_{\theta} \equiv \frac{\overline{u^{\prime} w^{\prime}} \frac{\partial \theta}{\partial z}}{\overline{w^{\prime} \theta^{\prime}} \frac{\partial U}{\partial z}}=\frac{\Phi_{h}}{\Phi_{m}}
$$

following the flux-gradient (Boussinesq) relations (2)-(5); the latter can be best considered as a modeler's choice.[21] The turbulent production or destruction due to effects of buoyancy, $B$, from equation (17), can then be written as

$$
B=-\frac{g}{\theta_{0}} \frac{\nu_{T}}{\sigma_{\theta}} \frac{\partial \theta}{\partial z}=-\nu_{T}\left(\frac{\partial U}{\partial z}\right)^{2} \frac{\zeta}{\sigma_{\theta}} \frac{\Phi_{h}}{\Phi_{m}^{2}},
$$

using equations (16), (17), (25) and (26). The expressions of $B$ from equation (27) are commonly used in literature.[5, 21, 22] In the present work, we will use the expression on the right-hand side of equation (27) for the $k-\varepsilon$ model for MOST. However, there is an inconsistency in unstable conditions, when the empirical similarity functions of equation (11) are substituted in equation (27), because a different relation for $\Phi_{B}$ is obtained compared with equation (17) $\left(\Phi_{B}=-\zeta \Phi_{m}\right.$ when using the constants of Dyer). The inconsistency is caused by the fact that the empirical similarity functions suggest that $\sigma_{\theta}=\Phi_{h} / \Phi_{m}^{2}$ in unstable conditions, which does not comply with equation (26).

\subsection{A consistent $k-\varepsilon$ model for MOST}

The standard $k-\varepsilon$ model is not consistent with MOST, which is shown in Section 3.1.1. An existing and a proposed method to solve the inconsistency with the standard $k-\varepsilon$ model are discussed in Sections 3.1.2 and 3.1.3, respectively.

\subsubsection{Inconsistency of MOST with the $k-\varepsilon$ equations}

The model equation for the TKE equation (21) can be simplified for the conditions corresponding to MOST, i.e.

$$
\mathcal{P}+B+\mathcal{D}_{k}-\varepsilon=0 .
$$

The transport of $\operatorname{TKE}\left(\mathcal{D}_{k}\right)$ that was modeled through diffusion in equation (22) can now be rewritten as

$$
\mathcal{D}_{k}=-\frac{C_{k \mathcal{D}}}{4} \frac{u_{*}^{3}}{\kappa L} \Phi_{\varepsilon}^{-3 / 2} \times \begin{cases}\Phi_{m}^{13 / 2} f_{\mathrm{un}}\left(\zeta, \gamma_{1}\right) & \zeta<0 \\ \Phi_{m}^{-7 / 2} f_{\mathrm{st}}(\zeta, \beta) & \zeta>0\end{cases}
$$

by employing equations (7), (11), (14), and (20) in order to be consistent with MOST. Here, $C_{\mathcal{D}_{k}}$ is a constant, and $f_{\text {un }}$ and $f_{\text {st }}$ are stability functions:

$$
\begin{aligned}
C_{k \mathcal{D}} & \equiv \frac{\kappa^{2}}{\sigma_{k} \sqrt{C_{\mu}}}, \\
f_{\mathrm{un}}\left(\zeta, \gamma_{1}\right) & \equiv(2-\zeta)+\frac{\gamma_{1}}{2}\left(1-12 \zeta+7 \zeta^{2}\right)-\frac{\gamma_{1}^{2}}{16} \zeta\left(3-54 \zeta+35 \zeta^{2}\right), \\
f_{\mathrm{st}}(\zeta, \beta) & \equiv(2-\zeta)-2 \beta \zeta(1-2 \zeta+2 \beta \zeta) .
\end{aligned}
$$

The budget of TKE for stable and unstable conditions is plotted in Figure 1 using the constants of Dyer (equation 12), where each individual term of equation (28) is plotted. In stable conditions, the diffusion term $\mathcal{D}_{k}$ is small compared to the production $\mathcal{P}$ and dissipation $\varepsilon$, consistent with the TKE profile from equation (20) being nearly constant with height. Note that the diffusion is negative near the wall (for $0<\zeta<\sim 0.1$ ), which might seem unphysical but is not problematic. Equation (28) holds because $\mathcal{P}-\varepsilon+B=0$ for $\sigma_{\theta}=1$, which means that a near equilibrium in TKE exist. In unstable conditions, the equation for TKE is not in equilibrium since the TKE profile from equation (20) varies with height, which results in a diffusion that is larger than the production and dissipation for $\zeta<-0.7$. Hence, it is not possible to maintain the TKE profile for unsteady atmospheric conditions using an unmodified $k$-equation.

Employing equation (21), the budget for the turbulent dissipation rate $\varepsilon$ corresponding to MOST can be written as

$$
\left(C_{\varepsilon, 1} \mathcal{P}-C_{\varepsilon, 2} \varepsilon+C_{\varepsilon, 3} B\right) \frac{\varepsilon}{k}+\mathcal{D}_{\varepsilon}=0,
$$




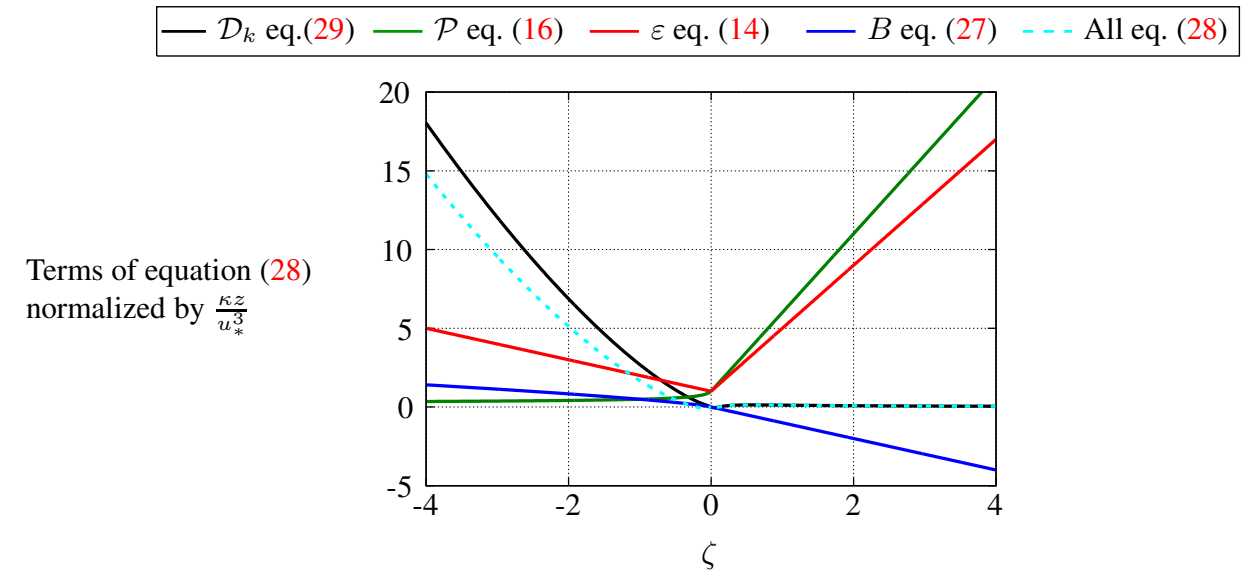

Figure 1. TKE budget for MOST, using the stability constants of Dyer of equation (12).

where the diffusion $\mathcal{D}_{\varepsilon}$, as defined in equation (22), reduces to

$$
\mathcal{D}_{\varepsilon}=\frac{u_{*}^{4}}{(\kappa z)^{2}} C_{k \mathcal{D}} \frac{\sigma_{k}}{\sigma_{\varepsilon}} C_{\mu}^{1 / 2} \Phi_{m}^{1 / 2} f_{\varepsilon}(\zeta)
$$

where

$$
f_{\varepsilon}(\zeta) \equiv\left\{\begin{array}{cc}
\Phi_{m}^{\frac{5}{2}}\left(1-\frac{3}{4} \gamma_{1} \zeta\right) & \zeta<0 \\
\Phi_{m}^{-\frac{5}{2}}\left(2 \Phi_{m}-1\right) & \zeta>0
\end{array}\right.
$$

The budget for $\varepsilon$ in MOST then becomes

$$
C_{\varepsilon, 1} \Phi_{m}-C_{\varepsilon, 2} \Phi_{\varepsilon}-C_{\varepsilon, 3} \zeta \frac{\Phi_{h}}{\sigma_{\theta} \Phi_{m}}+C_{k \mathcal{D}} \frac{\sigma_{k}}{\sigma_{\varepsilon}} \Phi_{\varepsilon}^{-\frac{1}{2}} f_{\varepsilon}(\zeta)=0
$$

The dimensionless MOST dissipation equation (36) reduces to equation (23) in neutral conditions $(\zeta=0)$. If equation (36) is satisfied, the MOST profiles are in balance with the $\varepsilon$-equation. This can be achieved by making $C_{\varepsilon, 3}$ a function of $\zeta$, which is discussed in the next two subsections.

\subsubsection{Method I: solution of Alinot and Masson}

Alinot[6] proposed to overcome the inconsistency of the $k$-equation with MOST by defining $\varepsilon$ explicitly as

$$
\varepsilon \equiv \mathcal{D}_{k}+\mathcal{P}+B
$$

while $\mathcal{D}_{k}, \mathcal{P}$ and $B$ are still computed with the $\varepsilon$-profile from MOST, as defined in equation (14). As a consequence, the $k$-equation is still not satisfied because $\varepsilon$ is not solved from equation (37). Nevertheless, Alinot and Masson[5] used the adapted $\varepsilon$, and they insert it into the $\varepsilon$-equation to derive a relation of $C_{\varepsilon, 3}^{*}$ as function of $\zeta$, where $C_{\varepsilon, 3}=C_{\varepsilon, 1}\left(1-C_{\varepsilon, 3}^{*}\right)$. Alinot and Masson[5] published a fifth order polynomial fit of the complex relation of $C_{\varepsilon, 3}^{*}$; however, Figure 2 shows that this fit is only valid for $-2.3<\zeta<2.0$ and their choice of model constants, as listed in Table I. The full relation of $C_{\varepsilon, 3}$ is equal to:

$$
C_{\varepsilon, 3}(\zeta)=\left\{\begin{array}{l}
\zeta^{-1}\left[C_{\varepsilon, 1}-C_{\varepsilon, 2}\left(\Phi_{\varepsilon}-\frac{5}{4} \kappa C_{k \mathcal{D}} \zeta \Phi_{m}^{\frac{11}{2}} \Phi_{\varepsilon}^{-\frac{3}{2}} g_{1}\right)+\frac{\kappa C_{k \mathcal{D}} \sigma_{k}}{\sigma_{\varepsilon}} \frac{\Phi_{m}^{-\frac{11}{2}} \Phi_{\varepsilon}^{\frac{7}{2}} g_{3}-\frac{5}{16} \kappa C_{k \mathcal{D}} \zeta^{2} g_{5}}{\Phi_{m}^{-11} \Phi_{\varepsilon}^{4}-\frac{5}{4} \kappa C_{k \mathcal{D}} \zeta \Phi_{m}^{-\frac{11}{2}} \Phi_{\varepsilon}^{\frac{3}{2}} g_{1}}\right] \\
\zeta<0 \\
\zeta^{-1}\left[C_{\varepsilon, 1} \Phi_{m}-C_{\varepsilon, 2}\left(\Phi_{\varepsilon}-\frac{1}{4} \kappa C_{k \mathcal{D}} \zeta \Phi_{m}^{-\frac{7}{2}} \Phi_{\varepsilon}^{-\frac{3}{2}} g_{2}\right)+\frac{\kappa C_{k \mathcal{D}} \sigma_{k}}{\sigma_{\varepsilon}} \frac{\Phi_{m}^{\frac{9}{2}} \Phi_{\varepsilon}^{\frac{7}{2}}\left(2 \Phi_{m}-1\right)+\frac{1}{16} \kappa C_{k \mathcal{D}} \zeta^{2} g_{4}}{\Phi_{m}^{7} \Phi_{\varepsilon}^{4}-\frac{1}{4} \kappa C_{k \mathcal{D}} \zeta \Phi_{m}^{\frac{7}{2}} \Phi_{\varepsilon}^{\frac{3}{2}} g_{2}}\right] \quad \zeta>0
\end{array}\right.
$$


using equation (30) and the following stability functions

$$
\begin{aligned}
& g_{1}=\frac{1}{5} f_{\text {un }}(\zeta, 16)=2-29 \zeta+184 \zeta^{2}-112 \zeta^{3}, \\
& g_{2}=f_{\text {st }}(\zeta, 5)=2-11 \zeta-80 \zeta^{2}, \\
& g_{3}=1-32 \zeta+316 \zeta^{2}, \\
& g_{4}=232-5008 \zeta-63959 \zeta^{2}-117600 \zeta^{3}+556800 \zeta^{4}+1536000 \zeta^{5}, \\
& g_{5}=104+2152 \zeta-13949 \zeta^{2}+18768 \zeta^{3}+144800 \zeta^{4}-1792 \zeta^{5}+1792 \zeta^{6} .
\end{aligned}
$$

The relation from equation (38) is valid for the stability coefficients of Dyer, given in equation (12). If the stability coefficients are kept implicit, the relation for $C_{\varepsilon, 3}$ becomes more cumbersome.

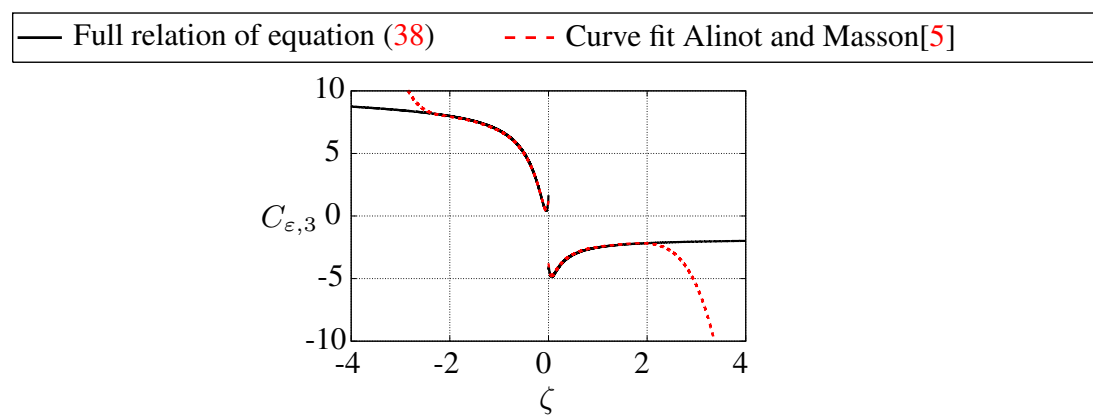

Figure 2. Method I: variable $C_{\varepsilon, 3}$. Full relation is plotted with the original model constants of Alinot and Masson[5], listed in Table I.

\subsubsection{Method II: new, proposed solution}

We propose to solve the incompatibility of the $k$-equation with MOST by introducing a source term $S_{k}$ in the $k$-equation

$$
\frac{D k}{D t}=\mathcal{D}_{k}+\mathcal{P}-\varepsilon+B-S_{k}
$$

where $S_{k}$ is defined as

$$
S_{k} \equiv \mathcal{D}_{k}+\mathcal{P}-\varepsilon+B=\frac{u_{*}^{3}}{\kappa L} \times \begin{cases}\zeta^{-1}\left(\Phi_{m}-\Phi_{\varepsilon}\right)-\frac{\Phi_{h}}{\sigma_{\theta} \Phi_{m}}-\frac{C_{k \mathcal{D}}}{4} \Phi_{m}^{13 / 2} \Phi_{\varepsilon}^{-3 / 2} f_{\mathrm{un}}\left(\zeta, \gamma_{1}\right) & \zeta<0 \\ 1-\frac{\Phi_{h}}{\sigma_{\theta} \Phi_{m}}-\frac{C_{k \mathcal{D}}}{4} \Phi_{m}^{-7 / 2} \Phi_{\varepsilon}^{-3 / 2} f_{\mathrm{st}}(\zeta, \beta) & \zeta>0\end{cases}
$$

using equations (30), (32) and (31). In stable atmospheric conditions, $S_{k}$ only counterbalances the small turbulent diffusion $\mathcal{D}_{k}$ for $\sigma_{\theta}=1$, and it is therefore small. $S_{k}$ is large in unstable atmospheric conditions because the diffusion is large and $\mathcal{P}-\varepsilon+B \neq 0$. The inconsistency in the $\varepsilon$-equation is solved with a parameterization of $C_{\varepsilon, 3}$, by using equation (36)

$$
C_{\varepsilon, 3}(\zeta)=\frac{\sigma_{\theta}}{\zeta} \frac{\Phi_{m}}{\Phi_{h}}\left(C_{\varepsilon, 1} \Phi_{m}-C_{\varepsilon, 2} \Phi_{\varepsilon}+\left[C_{\varepsilon, 2}-C_{\varepsilon, 1}\right] \Phi_{\varepsilon}^{-1 / 2} f_{\varepsilon}(\zeta)\right)
$$

where equation (23) is employed. $S_{k}$ and $C_{\varepsilon, 3}$ are plotted in Figure 3.
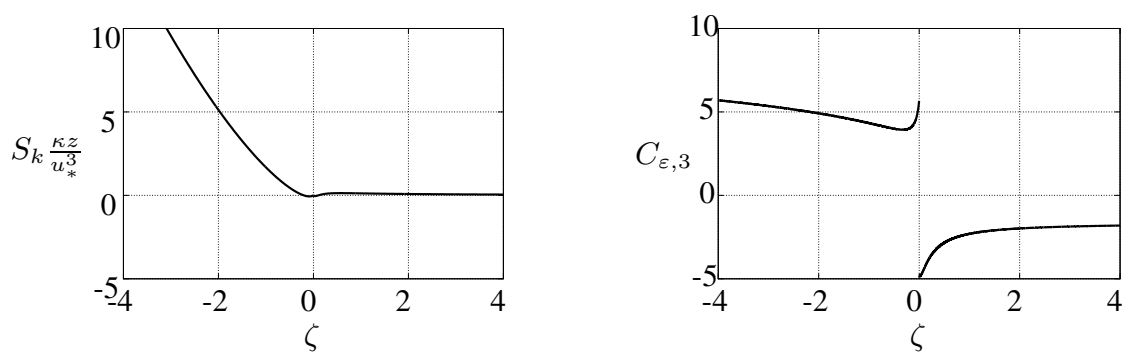

Figure 3. Method II: source term $S_{k}$ (equation (45)) and a variable $C_{\varepsilon, 3}$ (equation (46)).

Note that the typical wall boundary conditions for simulations of the neutral ASL using the $k-\varepsilon$ model[23] can still be used, because the MOST profiles approach neutral conditions at the wall, where $\zeta \rightarrow 0$. 


\section{SIMULATIONS}

\subsection{Methodology}

The incompressible finite volume code EllipSys3D is used.[19, 24] The Navier-Stokes equations are solved with the SIMPLE algorithm[25], and the QUICK scheme[26] is used to discretize the convective terms. Since the flow variables are stored in a co-located manner, decoupling of the pressure with body forces can occur. This problem is solved by a modified Rhie-Chow algorithm that is suitable for flows with body forces.[27, 28, 29]

The simulations are performed without solving a temperature equation, since the Bouyancy source term is not taken as a function of the potential temperature (right-hand side of equation (27)).

\subsubsection{Test cases}

In Table II, three test cases are defined that differ in atmospheric stability; a neutral, a stable $\left(\zeta_{\text {ref }}=0.5\right)$ and an unstable $\left(\zeta_{\text {ref }}=-0.5\right)$ case, where $\zeta_{\text {ref }}$ is the stability parameter at a reference height of $50 \mathrm{~m}$. A reference wind speed $U_{\text {ref }}$ of 10 $\mathrm{m} \mathrm{s}^{-1}$ is set for all cases, and a realistic reference turbulence intensity $I_{\text {ref }}$ of $0.08,0.12$ and 0.16 is chosen, for the stable, neutral and unstable cases, respectively. $I_{\text {ref }}$ is defined as

$$
I_{\mathrm{ref}} \equiv \frac{\sqrt{\frac{2}{3} k}}{U_{\mathrm{ref}}}=C_{\mu}^{-1 / 4} \frac{\kappa \sqrt{\frac{2}{3}}}{\ln \left(\frac{z_{\mathrm{ref}}}{z_{0}}\right)},
$$

which follows from equation (24).

In neutral conditions, $U_{\text {ref }}$ and $I_{\text {ref }}$ are set by the friction velocity $u_{*}$ and the roughness length $z_{0}$

$$
u_{*}=U_{\mathrm{ref}} I_{\mathrm{ref}} C_{\mu}^{1 / 4} \sqrt{\frac{3}{2}}, \quad z_{0}=z_{\mathrm{ref}} \exp \left(I_{\mathrm{ref}}^{-1} C_{\mu}^{-1 / 4} \kappa \sqrt{\frac{2}{3}}\right)
$$

using the logarithmic solution given in equation (24). In the non-neutral MOST simulations, $u_{*}$ and $z_{0}$ are set as

$$
\begin{aligned}
& u_{*}=U_{\mathrm{ref}} I_{\mathrm{ref}} C_{\mu}^{1 / 4} \sqrt{\frac{3}{2}} \Phi_{\varepsilon}^{-1 / 4}\left(\zeta_{\mathrm{ref}}\right) \Phi_{m}^{1 / 4}\left(\zeta_{\mathrm{ref}}\right), \\
& z_{0}=z_{\mathrm{ref}} \exp \left[-I_{\mathrm{ref}}^{-1} C_{\mu}^{-1 / 4} \kappa \sqrt{\frac{2}{3}} \Phi_{\varepsilon}^{1 / 4}\left(\zeta_{\mathrm{ref}}\right) \Phi_{m}^{-1 / 4}\left(\zeta_{\mathrm{ref}}\right)+\Psi_{m}\left(\zeta_{\mathrm{ref}}\right)\right] .
\end{aligned}
$$

The stability constants of Dyer from equation (12) are used in the MOST functions.

Table II. Test cases and corresponding parameters.

\begin{tabular}{lccccc}
\hline Case & $\begin{array}{c}\zeta_{\text {ref }} \text { at } 50 \mathrm{~m} \\
{[-]}\end{array}$ & $\begin{array}{c}U_{\text {ref }} \text { at } 50 \mathrm{~m} \\
{\left[\mathrm{~m} \mathrm{~s}^{-1}\right]}\end{array}$ & $\begin{array}{c}I_{\text {ref }} \text { at } 50 \mathrm{~m} \\
{[-]}\end{array}$ & $\begin{array}{c}z_{0} \\
{[\mathrm{~m}]}\end{array}$ & $\begin{array}{c}u_{*} \\
{\left[\mathrm{~m} \mathrm{~s}^{-1}\right]}\end{array}$ \\
\hline Stable & 0.5 & 10 & 0.06 & 0.0485 & 0.424 \\
Neutral & 0 & 10 & 0.10 & 0.0723 & 0.612 \\
Unstable & -0.5 & 10 & 0.20 & 0.0447 & 0.642
\end{tabular}

\subsubsection{Computational domain}

We would like to apply the proposed $k-\varepsilon$ model to non-neutral wake simulations in future work. Therefore, the computational domain that is used to test the capability of maintaining MOST inlet profiles is based on a domain suitable for single wake simulations, and it is shown in Figure 4. Inside the flow domain, a wake domain is defined in which a wind turbine represented as an actuator disk (AD)[30] could be placed. The wake domain has dimensions $12 D \times 3 D \times 2 D$ in x,y,z-direction, with $D=40 \mathrm{~m}$. In the wake domain, a uniform spacing of $D / 10$ is used in the horizontal directions, which is based on a grid study performed in previous work.[31] The first cell height is set to $0.1 \mathrm{~m}$ and the neighboring cells grow with height. Note that the cells do not exceed $D / 10$ inside the wake domain. The total amount of cells is around 1 million.

The width and height of the full domain is $16 \mathrm{D}$. The length of the domain is $22 D+x_{\mathrm{in}}$, where $x_{\mathrm{in}}$ is a parameter that is used to investigate the ability of maintaining the inflow profiles at the hypothetical AD location. In this study, $x_{\mathrm{in}}$ is set to 1 and $50 \mathrm{~km}$. The inflow profiles are inserted at the lateral boundary $x=0$ and at the horizontal boundary $z=16 D$. Symmetry conditions are imposed at the lateral boundaries at $y=0$ and $y=16 D$. An outlet is placed at $x=x_{\text {in }}+22 D$, at which a fully developed flow is assumed. At the wall boundary $z=0$, the turbulent dissipation is prescribed, while a Neumann condition is used for the TKE.[23] 


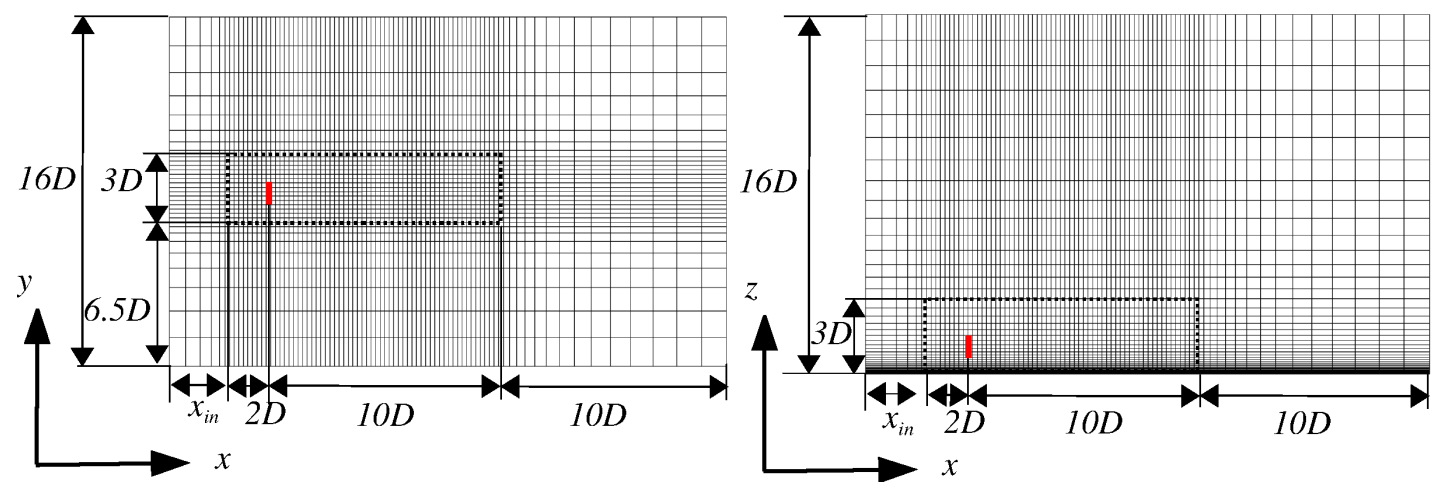

Figure 4. Computational domain with $x_{\mathrm{in}}=100 \mathrm{~m}$. Left: top view. Right: side view. Dashed black box marks the wake domain. Hypothetical AD is illustrated as a red filled box. One in every two nodes is shown.

\subsection{Results and Discussion}

In Section 3, it has been shown that $k$ - $\varepsilon$-equations of the standard $k-\varepsilon$ model are not in equilibrium with MOST, which means that MOST profiles are not sustained. Alinot and Masson[5] have proposed a modified $k-\varepsilon$ model for MOST, here labeled as the $k-\varepsilon$ MOST AM model, that uses a variable $C_{\varepsilon, 3}$ and a modified $\varepsilon$. We have proposed an alternative $k-\varepsilon$ model, labeled the $k-\varepsilon$ MOST model, where an analytical source term is introduced in the $k$-equation and an alternative variable $C_{\varepsilon, 3}$ is used. The $k-\varepsilon$ MOST and $k-\varepsilon$ MOST AM models are compared using a mesh that is suited for a single wind turbine wake, as shown in Figure 4, where $x_{\mathrm{in}}$ is 1 and $50 \mathrm{~km}$. The profiles at the hypothetical wind turbine location are extracted and compared with the inlet MOST profiles in Figures 5 and 6 for $x_{\text {in }}=1$ and $x_{\text {in }}=50 \mathrm{~km}$, respectively. Note that the $k$ - $\varepsilon$ MOST AM model is employed with the turbulence constants of the $k-\varepsilon$ MOST model, as listed in Table

\begin{tabular}{|c|c|c|}
\hline $\begin{aligned} & \text { Stable } \\
& \text { Inlet } \\
-\square- & k-\varepsilon \text { MOST } \\
-\Delta- & k-\varepsilon \text { MOST AM }\end{aligned}$ & $\begin{array}{ll} & \text { Neutral } \\
- & \text { Inlet } \\
-\square- & k-\varepsilon \text { MOST }\end{array}$ & $\begin{array}{cl} & \text { Unstable } \\
- & \text { Inlet } \\
-\square- & k-\varepsilon \text { MOST } \\
-\triangle- & k-\varepsilon \text { MOST AM }\end{array}$ \\
\hline
\end{tabular}
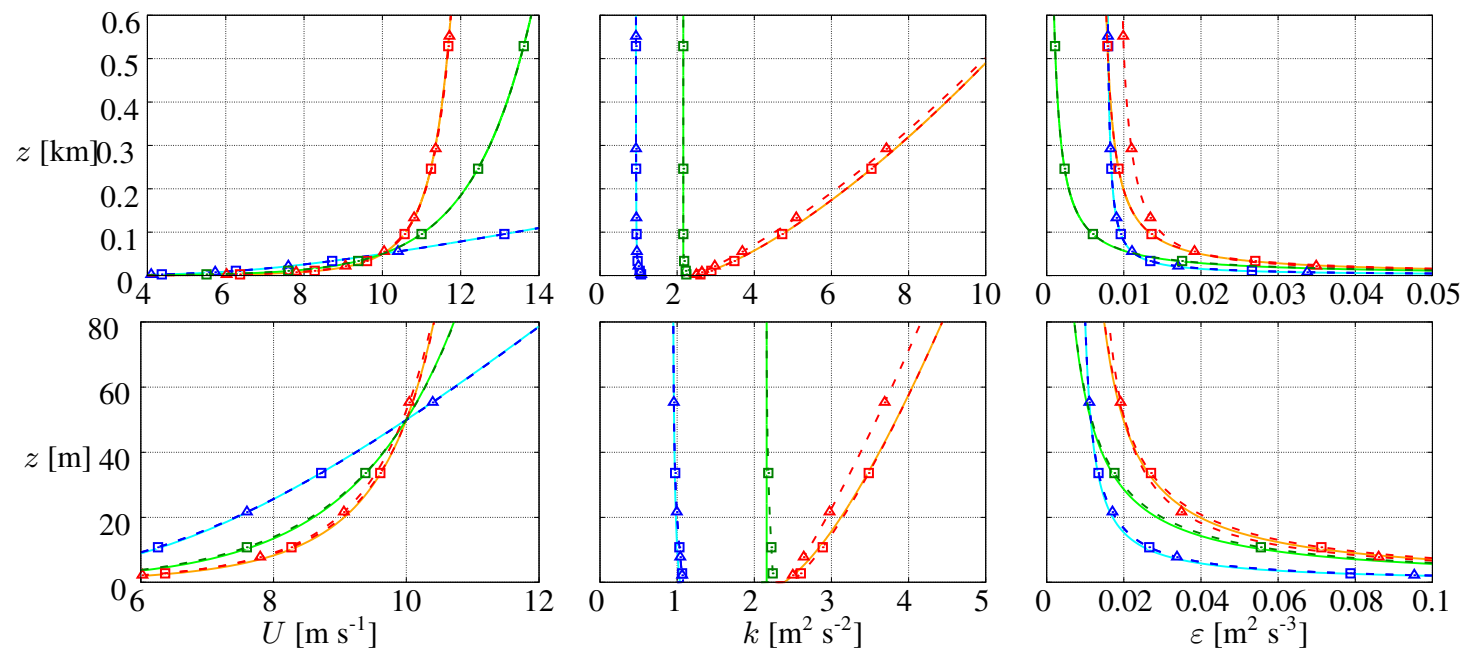

Figure 5. MOST profiles at the AD location, simulated in an empty domain using $x_{\mathrm{in}}=1 \mathrm{~km}$. The bottom plots are zoomed views of the top plots.

I. It is clear that $k-\varepsilon$ MOST AM model cannot sustain MOST profiles for unstable conditions over a long distance, because the $k$-equation is not in equilibrium with MOST, as discussed in Section 3.1.2. This results in an error in $k$ and $U$ of about $17 \%$ and $2 \%$ at the reference height of $50 \mathrm{~m}$, respectively, for $x_{\mathrm{in}}=50 \mathrm{~km}$. Even if the $k-\varepsilon$ MOST AM model is applied to a relative small domain $\left(x_{\mathrm{in}}=1 \mathrm{~km}\right)$, the error in $k$ and $U$, at the reference height, is still $7 \%$ and $1 \%$, respectively, as shown in Figure 6. The $k-\varepsilon$ MOST model can sustain MOST profiles because the additional source term keeps the $k$-equation in balance. For unstable conditions, the corresponding errors in $k$ and $U$ are smaller than $1 \%$ at the reference 


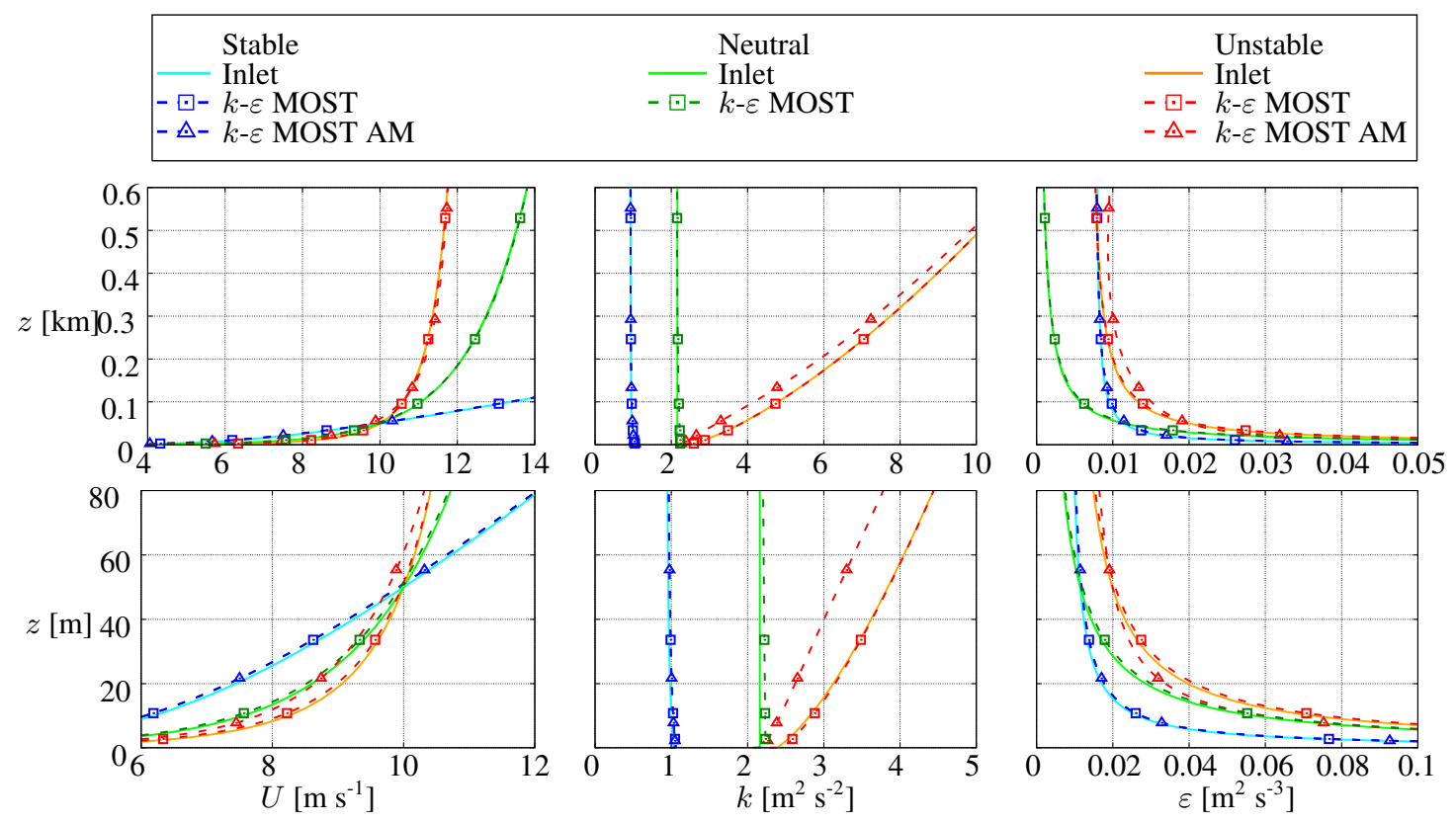

Figure 6. MOST profiles at the AD location, simulated in an empty domain using $x_{\mathrm{in}}=50 \mathrm{~km}$. The bottom plots are zoomed views of the top plots.

height. The remaining error is caused by the well known wall problem of $k$ at the wall.[32] In addition, the $k$ - $\varepsilon$ MOST model predicts the original $\varepsilon$ from MOST, while the $k-\varepsilon$ MOST AM model uses a modified $\varepsilon$ that shows large deviations with MOST.

\section{CONCLUSION}

The consistency of Monin-Obukhov similarity theory (MOST) with Reynolds-averaged Navier-Stokes (RANS) using the $k-\varepsilon$ model is investigated. The (non-neutral) MOST profiles are analytical functions that are not in equilibrium with the standard $k$ - $\varepsilon$-equations. It is shown that an additional source term in the $k$-equation (at least in unstable atmospheric conditions) and a variable $C_{\varepsilon, 3}$ can be used to sustain MOST profiles over a long distance of $50 \mathrm{~km}$. The $k$ - $\varepsilon$ model of Alinot and Masson[5] developed for MOST does not work well in unstable conditions, because it lacks the source term in the $k$-equation.

\section{ACKNOWLEDGEMENTS}

This work is supported by the Center for Computational Wind Turbine Aerodynamics and Atmospheric Turbulence funded by the Danish Council for Strategic Research, grant number 09-067216. Computational resources were provided by the DTU central computing facility.

\section{REFERENCES}

1. Svensson G, Holtslag AAM, Kumar V, Mauritsen T, Steeneveld GJ, Angevine E W Mand Bazile, Beljaars A, de Bruijn EIF, Cheng A, Conangla L, et al.. Evaluation of the diurnal cycle in the atmospheric boundary layer over land as represented by a variety of single-column models: The second GABLS experiment 2011; 140:177-206.

2. Motta M, Barthelmie RJ. The influence of non-logarithmic wind speed profiles on potential power output at Danish offshore sites. Wind Energy 2005; 8:219-236.

3. Monin AS, Obukhov AM. Basic laws of turbulent mixing in the surface layer of the atmosphere. Tr. Akad. Nauk. SSSR Geofiz. Inst. 1954 (translated by John Miller, 1959); 24:163-187. 
4. Freedman FR, Jacobson MZ. Modification of the standard $\epsilon$-equation for the stable ABL through enforced consistency with Monin-Obukhov similarity theory. Boundary-Layer Meteorology 2003; 106:383-410.

5. Alinot C, Masson C. $k-\varepsilon$ model for the atmospheric boundary layer under various thermal stratifications. Journal of Solar Energy Engineering 2005; 127:438-443.

6. Alinot C. Analyse aérodynamique des parcs éoliens immergés dans une couche limite terrestre ayant différentes conditions de stabilité thermique. PhD Thesis, École de Technologie Supérieure, Université du Québec, Montreal, Canada 2002.

7. Parente A, Corlé C, van Beeck J, Benocci C. Improved $k$ - $\varepsilon$ model and wall function formulation for the RANS simulation of ABL flows. Journal of Wind Engineering and Industrial Aerodynamics 2011; 99:267-278.

8. Panofsky HA, Dutton JA. Atmospheric Turbulence. Wiley-interscience, New York, US, 1984.

9. Monin AS, Yaglom AM. Statistical Fluid Mechanics, vol. 1. The MIT Press, 1971.

10. Högstrom U. Review of some basic characteristics of the atmospheric surface layer 1996; 78:215-246.

11. Boussinesq MJ. Théorie de l'écoulement tourbillonnant et tumultueux des liquides. Gauthier-Villars et fils, Paris, France, 1897.

12. Wyngaard JC. Toward numerical modeling in the 'Terra Incognita' 2004; 61:1816-1826.

13. Businger JA, Wyngaard JC, Izumi Y, Bradley EF. Flux-profile relationships in the atmospheric surface layer. Journal of Atmospheric Sciences 1971; 28:181-189.

14. Dyer AJ. A review of flux-profile relationships. Boundary-Layer Meteorology 1974; 7:363-372.

15. Högstrom U. Non-dimensional wind and temperature profiles in the atmospheric surface layer: A re-evaluation 1988; 29:55-78.

16. Kelly M, Larsen G, Dimitrov NK, Natarajan A. Probabilistic meteorological characterization for turbine loads. Journal of Physics: Conference Series 2014; 524(1):012 076.

17. Launder BE, Spalding DB. Mathematical models of turbulence. Academic Press, London, UK, 1972.

18. Wilcox DC. Turbulence Modeling for CFD. DWC Industries, 1994.

19. Sørensen NN. General purpose flow solver applied to flow over hills. PhD Thesis, Ris $\emptyset$ National Laboratory, Roskilde, Denmark 1994.

20. Richards PJ, Hoxey RP. Appropriate boundary conditions for computational wind engineering models using the $k$ - $\varepsilon$ turbulence model. Journal of Wind Engineering and Industrial Aerodynamics 1993; 46,47:145-153.

21. Sogachev A, Kelly M, Leclerc MY. Consistent two-equation closure modelling for atmospheric research: Buoyancy and vegetation implementations. Boundary-Layer Meteorology 2012; 145:307-327.

22. Prospathopoulos JM, Politis ES, Rados KG, Chaviaropoulos PK. Evaluation of the effects of turbulence model enhancements on wind turbine wake predictions. Wind Energy 2011; 14:285-300.

23. Sørensen NN, Bechmann A, Johansen J, Myllerup L, Botha P, Vinther S, Nielsen BS. Identification of severe wind conditions using a Reynolds Averaged Navier-Stokes solver. Journal of Physics: Conference series 2007; 75(012053):1-13.

24. Michelsen JA. Basis3d - a platform for development of multiblock PDE solvers. Technical Report AFM 92-05, Technical University of Denmark, Lyngby, Denmark 1992.

25. Patankar SV, Spalding DB. A calculation procedure for heat, mass and momentum transfer in three-dimensional parabolic flows. International Journal of Heat and Mass Transfer 1972; 15:1787-1806.

26. Leonard BP. A stable and accurate convective modelling procedure based on quadratic upstream interpolation. Computer Methods in Applied Mechanics and Engineering 1979; 19:59-98.

27. Troldborg N, Sørensen NN, Réthoré PE, van der Laan MP. A consistent method for finite volume discretization of body forces on collocated grids applied to flow through an actuator disk. Computers and Fluids 2015; 119:197-203.

28. Réthoré PE, Sørensen NN. A discrete force allocation algorithm for modelling wind turbines in computational fluid dynamics. Wind Energy 2012; 15:915-926.

29. Rhie CM, Chow WL. Numerical study of the turbulent fow past an airfoil with trailing edge separation. AIAA Journal 1983; 21:1525-1532.

30. Mikkelsen R. Actuator Disc Methods Applied to Wind Turbines. PhD Thesis, Technical University of Denmark, Mek dept, Lyngby, Denmark 2003.

31. van der Laan MP, Sørensen NN, Réthoré PE, Mann J, Kelly MC, Troldborg N, Schepers JG, Machefaux E. An improved $k-\varepsilon$ model applied to a wind turbine wake in atmospheric turbulence. Wind Energy May 2015; 18(5):889907, doi:10.1002/we.1736.

32. Blocken B, Stathopoulos T, Carmeliet J. CFD simulation of the atmospheric boundary layer: wall function problems. Atmospheric Environment 2007; 41:238-252. 